\title{
Introduction: Moyamoya Disease
}

\author{
R. Michael Scott, M.D., and Edward R. Smith, M.D. \\ Department of Neurosurgery, Children's Hospital, Boston, Massachusetts
}

\begin{abstract}
7 his issue of Neurosurgical Focus is devoted to the topic of moyamoya disease/syndrome. When the senior editor (R.M.S.) was a neurosurgical resident in the late 1960s and early 1970 s, the condition was virtually unknown in the Western hemisphere, and patients with "cerebrovascular insufficiency" and the typical arterial findings on angiography were believed to have a type of arteritis. The refinement of catheter angiography techniques and the development of the imaging modalities of CT and MR imaging clarified the significance of making the correct diagnosis of moyamoya disease in affected patients, and with the development of direct and then indirect revascularization procedures during this same period, neurosurgeons became involved in the disease's treatment.
\end{abstract}

After soliciting articles for this issue, we were struck by the broad range of submitted titles. Articles by J. Smith and Bendok et al. provide broad overviews of the disease and its manifestations. Steinberg et al. provide a thorough description of the information currently available on the origins and genetics of moyamoya disease. In other articles, Derdeyn et al., Lee et al., and Bacigaluppi et al. discuss the differing imaging and cerebral blood flow techniques that are available to evaluate the patient with moyamoya disease, and Spetzler et al. give a broad overview of the surgical techniques applicable in treating the condition. Czabanka et al. consider the relative efficacy of revascularization surgery in patient populations of different ages, a theme further amplified by Starke et al. in their evaluation of indirect versus direct operations in this population. Edward Smith reviews the literature on moyamoya syndrome seen in sickle cell disease and discusses the results of surgery in this patient population. The editors are grateful to the staff of the JNS Publishing Group for their help assembling and editing this issue of Neurosurgical Focus. 